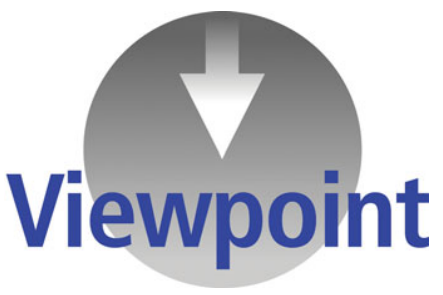

\title{
Molecular interaction networks in the analyses of sequence variation and proteomics data
}

\section{Ulrich Stelzl}

\author{
Max Planck Institute for Molecular Genetics (MPIMG), Otto-Warburg Laboratory, Berlin, Germany
}

Protein-protein interaction networks are typically generated in standard cell lines or model organisms as it is prohibitively difficult to record large interaction datasets from specific tissues or disease models at a reasonable pace. Although the interaction data are of high confidence, they thus do not reflect in vivo relationships as such. A wealth of physiologically relevant protein information, obtained under different conditions and from different systems, is available including information on genetic variation, protein levels, and PTMs. However, these data are difficult to assess comprehensively because the relationships between the entities remain elusive from the measurements. Here, we exemplarily highlight recent studies that gained deeper insight from genetic variation, protein, and PTM measurements using interaction information pointing toward the importance and potential of interaction networks for the interpretation of sequencing and proteomics data.

\section{Keywords:}

Genetic variation / MS / Phosphorylation / Posttranslational modification / Proteinprotein interactions / Yeast two-hybrid

Genotypes are expressed through regulated synthesis of encoded molecules, their activities and interactions. The resulting molecular networks involve most if not all molecules in the cell and their physical or functional relationship. Activity changes of genes and gene products are therefore propagated through the networks in the cell. Furthermore, the cellular network wiring is substantially modulated through all sorts of intracellular, extracellular, and environmental cues. Therefore, emergent properties of networks underlie phenotypes $[1,2]$. Even in its simplest form as nodes (representing molecules such as proteins, DNA, RNA, or metabolites) connected through edges (representing physical interaction or functional relationships), molecular networks represent some of the complexity of a cell [3]. Thus a vital research goal is to comprehensively record the molecules in cells, such

Correspondence: Dr. Ulrich Stelzl, Max Planck Institute for Molecular Genetics (MPIMG), Otto-Warburg Laboratory, Berlin, Germany

E-mail: stelzl@molgen.mpg.de

Abbreviations: AD, Alzheimer's disease; AP-MS, affinity purification coupled to MS; GWAS, genome-wide association studies; htt, huntingtin; PPI, protein-protein interaction; SNP, single nucleotide polymorphism; TF, transcription factor; $\mathbf{Y 2 H}$, yeast twohybrid
Received: June 3, 2013

Revised: August 2, 2013

Accepted: August 4, 2013 as RNAs, proteins with its various splice and modification states [4-7], if possible quantitatively under defined physiological conditions, and to systematically map the interactions between them [8-13].

Network properties and their correlation to disease are being exploited in many ways [14-17]. Because of the high connectedness and because the networks respond extensively to intra- and extra-cellular perturbations, the differences between molecular networks of defined states will generally be most informative for a better understanding of basic cellular processes [2] and, ultimately, of relevant alterations in a patient individual. Therefore, network-based approaches to human diseases focus on extracting differential networks, thereby stripping off the static, mainly housekeeping, information to capture the dynamic changes in cellular processes, or tissue and disease specific alterations in networks. Unless decent attempts will be made to consider comprehensive information of all molecules, i.e. built and learn to retrieve relevant information of whole cell network models [18,19], exploration of network modularity and disintegration of networks into predictive units are apparently successful strategies to pursue. Along this line, multiple network-based approaches linking comprehensive molecular data records such as genomic variation, RNA expression, proteomic or posttranslation modification profiles and pharmacological effects in a disease context have been put forward. These approaches are 


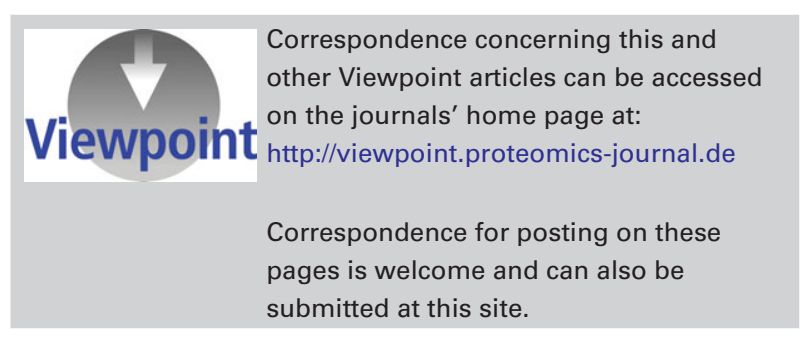

referred to as network medicine [14-17] or systems/network pharmacology [20-23] in the literature, with selected recent achievements pointed out here.

Expression profiles and proteomic measurements now record hundreds to thousands of differentially expressed genes or proteins from diverse tissues or specialized disease models. In analogy to mRNA expression data, the functional significance of long differential protein lists can be interpreted through gene set enrichment analysis methods. The methods statistically exploit precomputed annotations [24], pathways or more comprehensively protein neighborhoods [25] or protein complexes [26] to look for groups of genes with many more members than expected. These gene sets are then visualized as networks for better biological interpretation. With the power of modern MS to very quickly and deeply scan the differential proteomes of several tissues or organs, these scans may be useful in monitoring complex diseases or drug effects on an organismal level as altered gene sets of organs are a robust indication for dysfunction [27]. Although extremely useful, these methods strictly speaking do not use the network information in full, because the links do not contribute in the enrichment analyses. Proteins/genes are treated as balls in an urn and networks and pathway views are to display and ease the interpretation. The actual wiring displayed had had no direct impact on the analysis that led to the result. Not so in network-based approaches that exploit the relationships between the genes and proteins.

This principle can be illustrated by a study that established mouse transcription factor (TF) networks through mammalian two-hybrid interaction screens in a model cell line and measured TF expression levels across a panel of 54 human and mouse tissues. A 15 member subnetwork of interacting TFs [28] involved in tissue development was established through expression analysis also considering the differences in expression level across TF-TF interactions. This small subnetwork was best to discriminate the expression patterns of the cell lines very well separating tissue and stem cell types according to their embryological origin. In this approach, the six combinatorial interactions among TFs more than their expression levels alone were crucial to classify tissue identity. This suggests that relative small, however decisive, networks could suffice to distinguish relative complex phenotypes and that it is possible to extract them from highly connected global maps that have been generated under unrelated conditions in standard "systems biology" models.

Here, we > discuss selected studies that utilize genomics and proteomics data (recorded on network nodes) in combi- nation with interaction information, i.e. link information that is typically generated in "exogenous" systems, for a better understanding of cellular machines and disease-related cellular processes (Fig. 1). The underlying principle of all these approaches is that biologically relevant changes, often not the strongest signals in the data, cluster in networks. Therefore link information couples seemingly unrelated signals, which are then and only then, recognized because their dependency is considered.

Protein networks, in particular protein-protein interaction (PPI) networks, have quickly been recognized as systematic framework to search for and prioritize genes with genetic alterations in disease (Fig. 1A; for review see e.g. [14, 29, 30]). Essentially, genetic and epigenetic events related to a trait should somehow cluster in networks. With increase in coverage and accuracy of the networks that are used as a basis for the disease gene predictions, studies have revealed functionally more consistent prioritizations in different disease fields [30], including, e.g. neurodegeneration. The distance in a predicted physical human interaction network has been used to prioritize candidate genes from Alzheimer's disease (AD) genome-wide association studies (GWAS) and suggested potentially important specific linker genes [31]. Driven by interaction discovery experiments, a highly modular AD network was established with six GWAS candidate proteins directly interacting with proteins functionally characterized in $\mathrm{AD}$ [32]. In general, several network-based methods are available to prioritize mapped GWAS genes [33], however in principle networks can conversely aid GWAS hit identification. In networks called susceptibility modules [34], constructed from expression filtered PPI cliques, GWAS genes were enriched. Such a susceptibility PPI module was constructed to analyze seasonal allergic rhinitis-association and identified intragenic single nucleotide polymorphisms (SNPs) within 119 genes, which were 3.4 times more likely to be disease associated in a cohort of 4772 individuals and led to the identification of two new candidate genes [34].

In contrast to common genetic variants addressed in GWAS approaches, thousands to ten thousands of rare mutations and structural sequence variants in gene regions in each individual are found through whole genome/exome sequencing (Fig. 1A), a few of which will importantly contribute to a disease [35]. The importance and functional consequences of rare, sometimes even unique variants for a disease are difficult to assess statistically. Integrative network approaches have been employed to analyze the potential overall impact of rare variants in certain diseases, e.g. psychiatric disorders where GWAS did not yield many hits. In two studies Gilman et al. used a probabilistic human protein network to find clusters of proteins with rare SNPs and copy number variants in patients $[36,37]$. The subnetworks implicated in autism and schizophrenia were functionally related; however the associated genetic variants were likely functionally different for the different diseases [36]. Although consistent pathway signals were found, these analyses critically depend on the connectivity of 


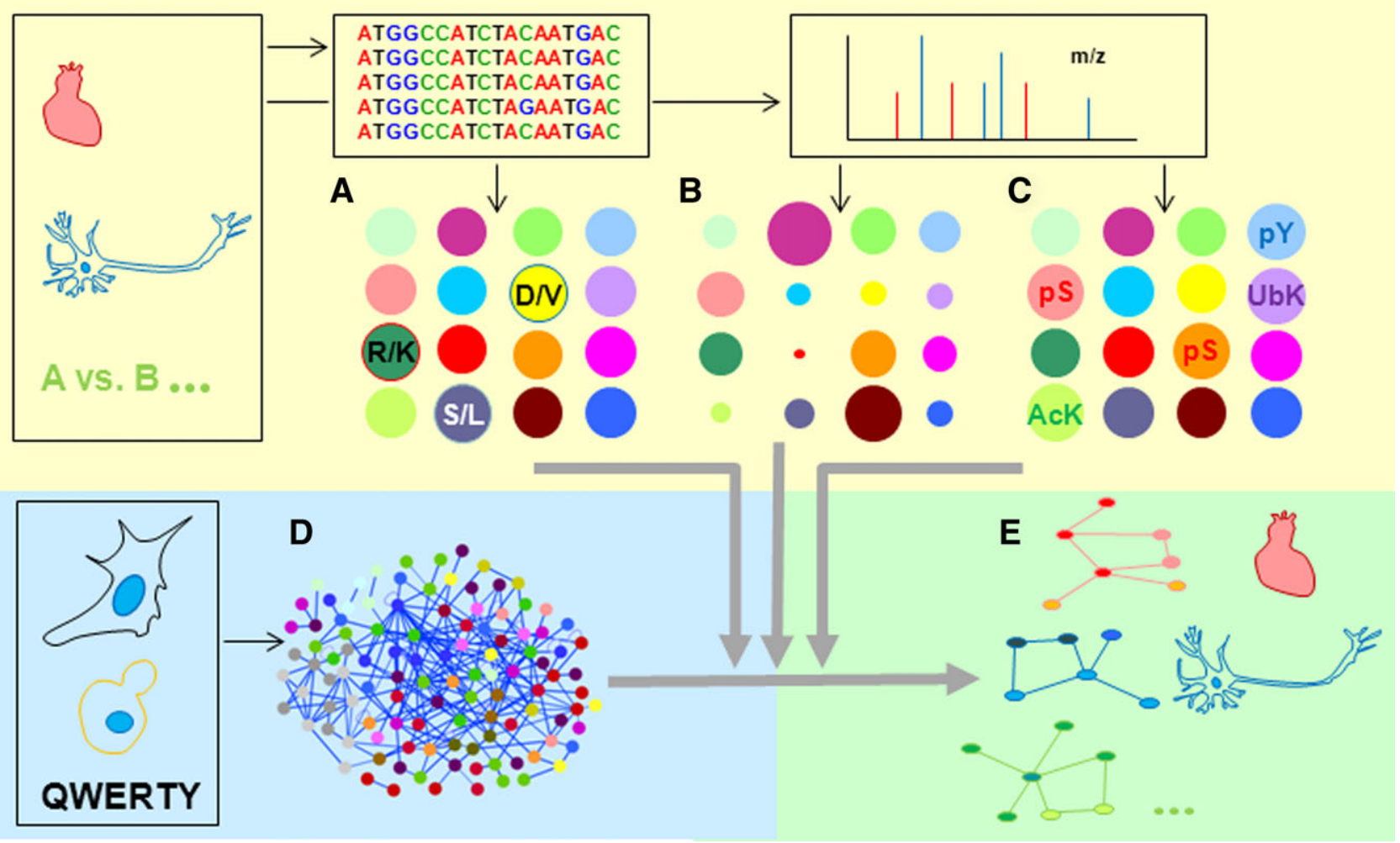

Figure 1. Molecular interaction information leads to a better interpretation of genomic and proteomic data. Node information such as patterns of genetic variation (A), differential protein levels and cocomplex membership (B) or changes in posttranslational protein modifications (C) can be measured with current sequencing and MS techniques in parallel from in vivo model systems, disease models, or patient samples (indicated by the insets representing a schematic work flow from in vivo models to sequence variation analysis and MS approaches, respectively). Interaction information is typically recorded in exogenous model systems through AP-MS or Y2H analysis (inset represents mammalian cell or yeast models) or inferred computationally (symbolized through standard keyboard signature in the inset) (D). The network information provides a very useful framework to integrate specific protein data resulting in highly informative differential networks (E) for different tissues, cellular processes or disease states.

proteins; i.e. the weighted sum of the edges is decisive for clustering.

A recent study in yeast reported SRM-based quantitative proteome measurements of individual yeast strains precise enough to map quantitative protein expression variation to single or epistatic genomic loci [7]. This suggests a proteomics approach examining individual variation, which is in principle applicable to humans $[38,39]$ and would lead toward a better understanding of the impact of genetic variation and genetic interactions on phenotypes.

A more mechanistic approach focuses on missense mutations that may alter interaction patterns of proteins. In a proof a principle study, several disease-related missense mutation give rise to differential protein interaction patterns [40] and disease-associated missense mutations clustered in interaction interfaces of proteins [41]. This is particularly striking when looking at genes that have several alleles linked to more than one disorder as interaction patterns (edgetic PPI profiles) [40] and clustering of mutations in distinct interaction interfaces [41] can explain the specificity of the disease phenotypes. TP63, e.g. harbors two distinct regions with mutations that are associated with ectrodactyly ectodermal dysplasia and ankyloblepharon ectodermal dysplasia, respectively. The first region is in the p53-like DNA-binding domain and the second region in the SAM2 domain, where mutations interfere with TP73 binding [40,41].

Relative protein levels are routinely obtained from label free or isotope-labeled quantification of protein samples from different conditions or from affinity purification of biological assemblies. Protein networks can be of very high value to better interpret and to gain more mechanistically understanding of protein enrichment data from these mass spec experiments (Fig. 1B). In particular, integration of differential protein list with networks (or vice versa) can provide inside into the dynamics of protein assemblies. For example, the spliceosomal machinery is now in the focus of disease research as cancer genome sequencing uncovered high frequency mutations in several spliceosomal genes [42]. Recently, a yeast two-hybrid $(\mathrm{Y} 2 \mathrm{H})$ network was generated providing more than 600 binary connections for the $\sim 240$ human spliceosomal proteins [43]. A set of 76 affinity purification experiments collected from a series of proteomics studies (reviewed in [44]) was converted 
into protein-protein interaction weights and used to cluster the $\mathrm{Y} 2 \mathrm{H}$ data into overlapping interaction modules [45] thus revealing dynamic interaction patterns. Central spliceosomal proteins and their specific interactions (e.g. with hPRP8) were identified that contribute to the exceptional compositional dynamics-and thus function-of this large ribonucleoprotein machine [43]. As a second example, also targeted in vivo affinity purification coupled to MS (AP-MS) data can provide deeper insight when analyzed as networks rather than a group of nodes or spoke expanded complexes. Twenty-four affinity purification experiments using huntingtin (htt), the polyglutamine containing protein causing Huntington's Disease, as a bait from a knock-in mouse model resulted a protein list of hundreds of candidate-binding proteins after standard filtering [46]. Protein lists of this size are a typical result for MS-based experiments and often show consistent pathway signatures. Shirasaki et al. then constructed weighted links between the $724 \mathrm{htt}$ co-purifying proteins by pairwise correlating peptide spectral counts over multiple conditions. The topological overlap, a network measure for common interaction partners of two proteins calculated from the pairwise correlation values, was used to cluster the data into eight htt-interactome modules, which were hierarchically related to distinct functional aspects such as brain region specificity, aging, and protein aggregation modulation or htt functions directly [46].

Over the years, MS-based proteomics has recorded more than 100000 PTMs, such as phosphorylation, ubiquitinylation, or acetylation, across two-thirds of the human proteome (Fig. 1C). Whether a PTM can be globally mapped is now largely dictated by the availability of affinity tools to enrich the modification in the samples. First studies are monitoring specific PTMs over dynamic processes such as quantitative measurements of phosophorylation levels over the cell cycle [6]. Phosphorylation dynamics can be clustered in different response behaviors, e.g. with respect to cell cycle or early and late behaviors and displayed on protein interaction networks [6, 47]. For example, an ataxia telangiectasia mutated kinase driven DNA-Damage phospho-response was put into context on the basis of PPI data, including nodes that have not been measured, reflecting the functional organization of the signaling in terms of protein complexes and functional network modules [47]. In another study, a directed protein interaction network was used to model epidermal growth factor (EGF) -dependent signal flow, i.e. EGF triggered phosphorylation events, in a time-dependent manner along outgoing links from the activated mitogen-activated protein kinase (MAPK) pathway members in a global PPI network [48].

Except for top-down proteomics approaches [49] and, very recently, sequential enrichment strategies [50], where detection of covariance in modification patterns is in sight, global modification measurement typically precludes the observation of combinatorial modifications. However, with large datasets of different PTMs at hand, the interplay of different types of PTMs can now be studied through data in- tegration, and network features are emergent. Two recent studies exploited the evolutionary conservation of PTMs revealing patterns of PTM cross-talk and modification coevolution and functionality $[51,52]$. All recorded modifications of an organism were merged and the conservation of the modification sites was assessed throughout phylogeny. Statistical significant associations of different sites revealed global networks of coevolving PTM types [51], likely cross-regulatory events between distinct types of modification on a given protein $[51,52]$, or important sites with potential effects on protein interaction and function [52]. In addition, a few studies are starting to assess the relationship between PTMs and protein interactions. The first large-scale study measuring lysineacetylation via MS has shown that acetylation accumulates over protein complexes in human cultured cells [53]. A computational analysis of global acetylation, phosphorylation, and ubiquitylation data revealed different levels of coordination of PTMs in protein interaction networks [54]. Hundreds of protein complexes selectively accumulating PTMs were identified together with protein regions of very high PTM densities. These regions, termed PTM integration spots, show domain-like properties and likely represent sites of high regulatory potential for signaling integration. Furthermore, in an analysis of phosphorylation sites in 26 large mitotic protein complexes that have been characterized via AP-MS [55], proteins, with more than four phospho-sites per 100 amino acids (switchboard subunits), were observed. A recent study also suggested that phosphorylation-associated SNPs in cancer genomes may play assorted roles [56]. Together these studies strongly emphasize network-based dataset integration as requisite in proteomic PTM studies to better predict modification impact on cellular function.

Individual genome sequencing [35] is now reliably recording rare genetic variations in gene regions. Furthermore, expression profiling and RNA-seq comprehensively informs about the mRNA repertoire of the cell [4], while MS-based full proteome measurements become quantitative [5] and targeted [57] and protein modification sites can be measured at a large scale $[6,53]$. As fast and promising these developments are, PPI techniques are lacking that comprehensively measure the presence, absence, or strength of the interactions between these molecules in parallel even for a single defined state or condition. For now, protein interaction networks are recorded exogenously in model systems ( [9-13], Fig. 1D) and only a few methods exist at all that can measure interactions in intact (fixed) cells or tissues. These methods include proximity ligation assays [58], protein complementation assays [59] and cross-linking proteomic techniques [60]. The latter technique, which tries to reliably identify cross-linked peptides via MS, has been used to map interactions within and between subunits of RNA polymerase II [61] or protein phosphatase 2A [62] and led to better structural interpretation of these large assemblies. In theory, one could think of shotgun approaches using cells treated with a cross-linking agent as input [60]. Currently, generic high quality protein networks generated through large-scale 
screening techniques such as $\mathrm{Y} 2 \mathrm{H}$ or AP-MS provide a major resource for interpretation of genetic, expression, proteome, and protein modification data. The key tasks are to integrate specific genome/proteome measurements, assess connectivity and separate cellular processes or disease processes on the network level. Specific subnetworks (Fig. 1E) will provide the set of linked molecules that can be measured quantitatively for the corresponding conditions, tissues, organs, and ultimately in samples of individuals. In principle, current work suggests that network guided, relatively small sets of quantitative measurements recorded from individuals would already be quite powerful in informing models for a more personalized treatment of diseases.

The author thanks the current members of the lab for fruitful discussions and Dr. Jonathan Woodsmith for critical reading of the manuscript. The work in the lab was supported by the MaxPlanck Society.

The author has declared no conflict of interest.

\section{References}

[1] Vidal, M., Cusick, M. E., Barabasi, A. L., Interactome networks and human disease. Cell 2011, 144, 986-998.

[2] Ideker, T., Krogan, N. J., Differential network biology. Mol. Syst. Biol. 2012, 8, 565.

[3] Barabasi, A. L., The network takeover. Nat. Phys. 2012, 8, 14-16.

[4] Sultan, M., Schulz, M. H., Richard, H., Magen, A. et al., A global view of gene activity and alternative splicing by deep sequencing of the human transcriptome. Science 2008, 321, 956-960.

[5] Schwanhausser, B., Busse, D., Li, N., Dittmar, G. et al., Global quantification of mammalian gene expression control. $\mathrm{Na}$ ture 2011, 473, 337-342.

[6] Olsen, J. V., Vermeulen, M., Santamaria, A., Kumar, C. et al., Quantitative phosphoproteomics reveals widespread full phosphorylation site occupancy during mitosis. Sci. Signal. 2010, 3, ra3.

[7] Picotti, P., Clement-Ziza, M., Lam, H., Campbell, D. S. et al., A complete mass-spectrometric map of the yeast proteome applied to quantitative trait analysis. Nature 2013, 494, 266270.

[8] Stelzl, U., Worm, U., Lalowski, M., Haenig, C. et al., A human protein-protein interaction network: a resource for annotating the proteome. Cell 2005, 122, 957-968.

[9] Venkatesan, K., Rual, J. F., Vazquez, A., Stelzl, U. et al., An empirical framework for binary interactome mapping. Nat. Methods 2009, 6, 83-90.

[10] Yu, H., Tardivo, L., Tam, S., Weiner, E. et al., Next-generation sequencing to generate interactome datasets. Nat. Methods 2011, 8, 478-480.

[11] Weimann, M., Grossmann, A., Woodsmith, J., Ozkan, Z. et al., A $\mathrm{Y} 2 \mathrm{H}$-seq approach defines the human protein methyltransferase interactome. Nat. Methods 2013, 10, 339-342.
[12] Havugimana, P. C., Hart, G. T., Nepusz, T., Yang, H. et al., A census of human soluble protein complexes. Cell 2012, 150, 1068-1081.

[13] Malovannaya, A., Lanz, R. B., Jung, S. Y., Bulynko, Y. et al., Analysis of the human endogenous coregulator complexome. Cell 2011, 145, 787-799.

[14] Barabasi, A. L., Gulbahce, N., Loscalzo, J., Network medicine: a network-based approach to human disease. Nat. Rev. Genet. 2011, 12, 56-68.

[15] Pawson, T., Linding, R., Network medicine. FEBS Lett. 2008, $582,1266-1270$

[16] Zanzoni, A., Soler-Lopez, M., Aloy, P., A network medicine approach to human disease. FEBS Lett. 2009, 583, $1759-1765$.

[17] Silverman, E. K., Loscalzo, J., Network medicine approaches to the genetics of complex diseases. Discov. Med. 2012, 14, 143-152.

[18] Zazzu, V., Regierer, B., Kuhn, A., Sudbrak, R., Lehrach, H., IT future of medicine: from molecular analysis to clinical diagnosis and improved treatment. N. Biotechnol. 2012, 30, 362-365.

[19] Califano, A., Striking a balance between feasible and realistic biological models. Sci. Transl. Med. 2011, 3, 103ps139.

[20] Hopkins, A. L., Network pharmacology. Nat. Biotechnol. 2007, 25, 1110-1111.

[21] Zhao, S., lyengar, R., Systems pharmacology: network analysis to identify multiscale mechanisms of drug action. Annu. Rev. Pharmacol. Toxicol. 2012, 52, 505-521.

[22] Hasan, S., Bonde, B. K., Buchan, N. S., Hall, M. D., Network analysis has diverse roles in drug discovery. Drug Discov. Today 2012, 17, 869-874.

[23] Csermely, P., Korcsmaros, T., Kiss, H. J., London, G., Nussinov, R., Structure and dynamics of molecular networks: a novel paradigm of drug discovery: a comprehensive review. Pharmacol. Ther. 2013, 138, 333-408.

[24] Merico, D., Isserlin, R., Stueker, O., Emili, A., Bader, G. D., Enrichment map: a network-based method for gene-set enrichment visualization and interpretation. PLoS One 2010, 5 , e13984.

[25] Kamburov, A., Stelzl, U., Lehrach, H., Herwig, R., The ConsensusPathDB interaction database: 2013 update. Nucleic Acids Res. 2013, 41, D793-D800.

[26] Vinayagam, A., Hu, Y., Kulkarni, M., Roesel, C. et al., Protein complex-based analysis framework for high-throughput data sets. Sci. Signal. 2013, 6, rs5.

[27] Meierhofer, D., Weidner, C., Hartmann, L., Mayr, J. A. et al., Protein sets define disease states and predict in vivo effects of drug treatment. Mol. Cell Proteomics 2013, 12, M112.025031.

[28] Ravasi, T., Suzuki, H., Cannistraci, C. V., Katayama, S. et al., An atlas of combinatorial transcriptional regulation in mouse and man. Cell 2010, 140, 744-752.

[29] Piro, R. M., Network medicine: linking disorders. Hum. Genet. 2012, 131, 1811-1820.

[30] Califano, A., Butte, A. J., Friend, S., Ideker, T., Schadt, E., Leveraging models of cell regulation and GWAS data in 
integrative network-based association studies. Nat. Genet. 2012, 44, 841-847.

[31] Elefsinioti, A., Sarac, O. S., Hegele, A., Plake, C. et al., Largescale de novo prediction of physical protein-protein association. Mol. Cell Proteomics 2011, 10, M111.010629.

[32] Soler-Lopez, M., Zanzoni, A., Lluis, R., Stelzl, U., Aloy, P., Interactome mapping suggests new mechanistic details underlying Alzheimer's disease. Genome Res. 2011, 21, 364-376.

[33] Guney, E., Oliva, B., Exploiting protein-protein interaction networks for genome-wide disease-gene prioritization. PLOS One 2012, 7, e43557.

[34] Barrenas, F., Chavali, S., Alves, A. C., Coin, L. et al., Highly interconnected genes in disease-specific networks are enriched for disease-associated polymorphisms. Genome Biol. 2012, 13, R46.

[35] Abecasis, G. R., Auton, A., Brooks, L. D., DePristo, M. A. et al., An integrated map of genetic variation from 1,092 human genomes. Nature 2012, 491, 56-65.

[36] Gilman, S. R., Chang, J., Xu, B., Bawa, T. S. et al., Diverse types of genetic variation converge on functional gene networks involved in schizophrenia. Nat. Neurosci. 2012, 15, 1723-1728.

[37] Gilman, S. R., lossifov, I., Levy, D., Ronemus, M. et al., Rare de novo variants associated with autism implicate a large functional network of genes involved in formation and function of synapses. Neuron 2011, 70, 898-907.

[38] Johansson, A., Enroth, S., Palmblad, M., Deelder, A. M. et al., Identification of genetic variants influencing the human plasma proteome. Proc. Natl. Acad. Sci. USA 2013, 110, 4673-4678.

[39] Wu, L., Candille, S. I., Choi, Y., Xie, D. et al., Variation and genetic control of protein abundance in humans. Nature 2013, 499, 79-82.

[40] Zhong, Q., Simonis, N., Li, Q. R., Charloteaux, B. et al., Edgetic perturbation models of human inherited disorders. Mol. Syst. Biol. 2009, 5, 321.

[41] Wang, X., Wei, X., Thijssen, B., Das, J. et al., Threedimensional reconstruction of protein networks provides insight into human genetic disease. Nat. Biotechnol. 2012, 30, 159-164.

[42] Garraway, L. A., Lander, E. S., Lessons from the cancer genome. Cell 2013, 153, 17-37.

[43] Hegele, A., Kamburov, A., Grossmann, A., Sourlis, C. et al., Dynamic protein-protein interaction wiring of the human spliceosome. Mol. Cell 2012, 45, 567-580.

[44] Wahl, M. C., Will, C. L., Luhrmann, R., The spliceosome: design principles of a dynamic RNP machine. Cell 2009, 136, 701-718.

[45] Kamburov, A., Grossmann, A., Herwig, R., Stelzl, U., Clusterbased assessment of protein-protein interaction confidence. BMC Bioinformatics 2012, 13, 262.

[46] Shirasaki, D. I., Greiner, E. R., Al-Ramahi, I., Gray, M. et al.,
Network organization of the huntingtin proteomic interactome in mammalian brain. Neuron 2012, 75, 41-57.

[47] Bensimon, A., Schmidt, A., Ziv, Y., Elkon, R. et al., ATMdependent and -independent dynamics of the nuclear phosphoproteome after DNA damage. Sci. Signal. 2010, 3, rs3.

[48] Vinayagam, A., Stelzl, U., Foulle, R., Plassmann, S. et al., A directed protein interaction network for investigating intracellular signal transduction. Sci. Signal. 2011, 4, rs8.

[49] Tran, J. C., Zamdborg, L., Ahlf, D. R., Lee, J. E. et al., Mapping intact protein isoforms in discovery mode using top-down proteomics. Nature 2011, 480, 254-258.

[50] Swaney, D. L., Beltrao, P., Starita, L., Guo, A. et al., Global analysis of phosphorylation and ubiquitylation cross-talk in protein degradation. Nat. Methods 2013, 10, 676-682.

[51] Minguez, P., Parca, L., Diella, F., Mende, D. R. et al., Deciphering a global network of functionally associated posttranslational modifications. Mol. Syst. Biol. 2012, 8, 599.

[52] Beltrao, P., Albanese, V., Kenner, L. R., Swaney, D. L. et al., Systematic functional prioritization of protein posttranslational modifications. Cell 2012, 150, 413-425.

[53] Choudhary, C., Kumar, C., Gnad, F., Nielsen, M. L. et al., Lysine acetylation targets protein complexes and co-regulates major cellular functions. Science 2009, 325, 834-840.

[54] Woodsmith, J., Kamburov, A., Stelzl, U., Dual coordination of post translational modifications in human protein networks. PLoS Comput. Biol. 2013, 9, e1002933.

[55] Hegemann, B., Hutchins, J. R., Hudecz, O., Novatchkova, M. et al., Systematic phosphorylation analysis of human mitotic protein complexes. Sci. Signal. 2011, 4, rs12.

[56] Reimand, J., Bader, G. D., Systematic analysis of somatic mutations in phosphorylation signaling predicts novel cancer drivers. Mol. Syst. Biol. 2013, 9, 637.

[57] Picotti, P., Aebersold, R., Selected reaction monitoring-based proteomics: workflows, potential, pitfalls and future directions. Nat. Methods 2012, 9, 555-566.

[58] Weibrecht, I., Leuchowius, K. J., Clausson, C. M., Conze, T. et al., Proximity ligation assays: a recent addition to the proteomics toolbox. Expert. Rev. Proteomics 2010, 7, 401-409.

[59] Michnick, S. W., Ear, P. H., Manderson, E. N., Remy, I., Stefan, E., Universal strategies in research and drug discovery based on protein-fragment complementation assays. Nat. Rev. Drug Discov. 2007, 6, 569-582.

[60] Rappsilber, J., Cross-linking/mass spectrometry as a new field and the proteomics information mountain of tomorrow. Expert Rev. Proteomics 2012, 9, 485-487.

[61] Chen, Z. A., Jawhari, A., Fischer, L., Buchen, C. et al., Architecture of the RNA polymerase II-TFIIF complex revealed by cross-linking and mass spectrometry. EMBO J. 2010, 29, 717-726.

[62] Herzog, F., Kahraman, A., Boehringer, D., Mak, R. et al., Structural probing of a protein phosphatase $2 \mathrm{~A}$ network by chemical cross-linking and mass spectrometry. Science 2012, 337, 1348-1352. 